\title{
Modelos de Disponibilidade para Nuvens Privadas: Rejuvenescimento de Software Habilitado por Agendamento de Migração de VMs
}

\author{
Matheus D'Eça Torquato de Melo ${ }^{1}$, Paulo Romero Martins Maciel (Orientador) ${ }^{2}$
}

\author{
${ }^{1}$ Instituto Federal do Sertão Pernambucano - Campus Salgueiro \\ Salgueiro - PE, Brasil \\ ${ }^{2}$ Centro de Informática (CIn) - Universidade Federal de Pernambuco \\ Recife - PE, Brasil. \\ matheus.torquato@ifsertao-pe.edu.br, prmmecin.ufpe.br
}

\begin{abstract}
Reliability and availability are some of main barriers to managers migrate their applications to cloud computing environments. In this context, software aging rises as a potential problem, because it affects reliability directly through a gradual degradation which is difficult to detect. This Master Dissertation brings a set of analytical models for availability assessment on private clouds with software rejuvenation enabled by live VM Migration. The main purpose is to present proper schedules of migration in order to maximize system availability. The study was conducted in a way to improve final models. Experiments were conducted on real platforms, their results are used as basis to final models. Among the contributions we can highlight: methodology to availability models validation, approach to conduct software aging and rejuvenation experiments, and a set of analytical models to evaluate availability on private clouds with aspects of software aging and rejuvenation.
\end{abstract}

Resumo. Confiabilidade e disponibilidade são alguns dos principais desafios para administradores migrarem suas aplicações para ambientes de computação em nuvem. Nesse contexto, o envelhecimento de software surge como um problema potencial, pois afeta diretamente a confiabilidade do sistema através de uma degradação gradual e de difícil detecção. Essa dissertação traz um conjunto de modelos analíticos para avaliação de disponibilidade em ambientes de computação em nuvem privados com um mecanismo de rejuvenescimento de software suportado por migração de máquinas virtuais. O principal propósito é propor agendamentos adequados de migrações para maximizar a disponibilidade do ambiente. O estudo foi conduzido de modo a fortalecer os modelos finais, sendo assim conduzidos experimentos em plataformas reais prévios para servir de insumo aos modelos finais. Dentre as contribuições dadas pelo trabalho destacam-se: metodologia para validação de modelos de disponibilidade, metodologia para condução de experimentos de envelhecimento de software, modelos analíticos para avaliação de disponibilidade em nuvens privadas considerando aspectos de envelhecimento e rejuvenescimento de software.

Tipo de trabalho: Dissertação de mestrado.

Departamento: Centro de Informática (CIn), Universidade Federal de Pernambuco (UFPE).

Data da defesa: 10 de março de 2014.

URL: http://www.modcs.org/wp-content/uploads/thesis/Dissertacao-Matheus.pdf 


\section{Introdução}

Confiabilidade e disponibilidade aparecem como um dos maiores desafios para que as empresas e organizações migrem suas aplicações de ambientes de computação em nuvem [CISCO 2012]. Algumas pesquisas mostram que os ambientes de computação em nuvem sofrem com o envelhecimento de software [Araujo et al. 2011], problema que afeta diretamente a disponibilidade e confiabilidade dos sistemas. O envelhecimento de software se manifesta como uma diminuição gradual na confiabilidade do software conforme seu tempo de atividade [Huang et al. 1995]. Os efeitos relativos ao envelhecimento ocorrem devido à acumulação de erros e bugs enfrentados durante a execução. $\mathrm{O}$ acúmulo desses efeitos leva o sistema à travamentos, perda de desempenho e até falhas generalizadas [Grottke et al. 2008]. A detecção de efeitos relativos ao envelhecimento de software constitui uma tarefa árdua para administradores de sistemas, pois, geralmente, esses efeitos ocorrem após intervalos de tempo incertos (normalmente longos) [Melo et al. 2013].

A contramedida em relação aos efeitos de envelhecimento de software é denominada de rejuvenescimento de software. O rejuvenescimento de software consiste em reconduzir a aplicação para um estado livre do acúmulo de problemas acarretados pelo envelhecimento de software [Huang et al. 1995]. As operações de rejuvenescimento geralmente são baseadas no reboot do sistema operacional ou no restart das aplicações que sofrem com efeitos de envelhecimento de software [Grottke et al. 2008]. Porém, no contexto da disponibilidade e confiabilidade de sistemas, mesmo a utilização de operações de rejuvenescimento de software podem ser prejudiciais, pois acarretam a paralisação no sistema em execução [Matias et al. 2006]. Portanto, é necessário alcançar agendamentos ideais de rejuvenescimento de software para maximizar a disponibilidade do sistema.

Para checar a efetividade de mecanismos de rejuvenescimento de software é necessário fazer a avaliação de desempenho do sistema observado, para averiguar se os efeitos de envelhecimento de software foram realmente dirimidos após a realização da operação de rejuvenescimento de software. Existem três métodos principais para avaliar o desempenho dos sistemas: a medição, a simulação e a modelagem [Jain 1991]. Dentre essas, a modelagem consiste em avaliar o desempenho do sistema a partir de uma descrição formal (modelo) de seu funcionamento. Dentre as vantagens na utilização da modelagem como mecanismo de avaliação de desempenho estão as seguintes: possibilidade de realizar a avaliação sem interferência direta no sistema, avaliar o sistema antes de sua implementação, avaliar diferentes cenários a partir de um único modelo. Porém, na avaliação de desempenho baseada em modelagem, é necessário lidar com aproximações numéricas e valores estimados.

A modelagem também é utilizada para avaliação de dependabilidade de sistemas [Maciel et al. 2012]. Haja vista que os eventos que afetam a dependabilidade de sistemas (falhas, interrupções) ocorrem de maneira inesperada, a modelagem torna-se uma abordagem adequada pois permite estimar a dependabilidade dos sistemas sem a necessidade de medições realizadas em ambiente real.

A dissertação apresentada tem como objetivo principal propor um conjunto de modelos analíticos para avaliação de disponibilidade em ambientes de computação em nuvem que consideram o mecanismo de live migration como suporte ao rejuvenescimento de software. A avaliação é realizada para obter políticas adequadas para cada cenário estudado, visando maximizar a disponibilidade estacionária do sistema. 
De modo mais específico, para alcançar o objetivo principal, este trabalho propõese a: (i) Definir modelos de disponibilidade para plataformas de computação em nuvem que apresentam efeitos de envelhecimento de software (Seção 4.2 da dissertação); (ii) Agregar o comportamento de rejuvenescimento habilitado por agendamento de migração aos modelos de avaliação de disponibilidade (Seção 4.2 da dissertação); (iii) Avaliar diferentes políticas de rejuvenescimento de software em busca da mais adequada para cada cenário estabelecido (Seção 5.1 da dissertação).

Para robustecer o estudo foram estabelecidas atividades secundárias com o intuito de embasar os modelos gerados para alcançar o objetivo principal. São estas: (i) Realizar estudos experimentais em ambientes de computação em nuvem, visando encontrar os indícios de envelhecimento (Capítulo 3 da dissertação); (ii) Determinar a efetividade de mecanismos de rejuvenescimento para dirimir os efeitos causados pelo envelhecimento (Capítulo 3 da dissertação); (iii) Propor e validar modelos para infraestruturas básicas de computação em nuvem, a fim de serem utilizados como insumo para o aprofundamento dos estudos, que consideram o envelhecimento de software (Seções 4.1 e 5.1 da dissertação); (iv) Estudar a relevância das interrupções causadas por cada migração para a disponibilidade estacionária do sistema (Seção 5.4 da dissertação).

\section{Visão geral}

Esta seção apresenta uma visão geral das atividades e métodos adotados para alcançar os objetivos propostos pela dissertação. Dividiu-se a metodologia em ações menores com objetivos específicos, que resultam em insumos para a produção dos modelos finais.

A primeira atividade pertinente é realizar experimentos em uma plataforma de nuvem real, visando encontrar indícios de envelhecimento que justifiquem a construção dos modelos. Por isso, foram realizados experimentos com estresse acelerado e monitoramento de recursos em uma plataforma de nuvem privada OpenNebula visando observar o aparecimento de indícios de envelhecimento de software. Além disso devem ser realizados experimentos para checar a efetividade do mecanismo de rejuvenescimento proposto.

A metodologia de rejuvenescimento deve ser adequada para o envelhecimento de software observado no ambiente. O método selecionado foi o de rejuvenescimento de software habilitado por migração de máquinas virtuais. A migração consiste em mover a $V M$ da máquina física que a hospeda (máquina source) para outra máquina física (máquina target). Assim, a VM que está no sistema que acumula efeitos de envelhecimento (no caso, a máquina source) é migrada para outra que, no momento, está livre de efeitos de envelhecimento. Logo que a VM é completamente deslocada para outra máguina física, a máquina source, que está com efeitos de envelhecimento acumulado, pode ser rejuvenescida sem interromper o sistema que está sendo disponibilizado pela VM.

Então, os experimentos foram realizados com três fases específicas: $1^{\circ}$ - Estresse (para sobrecarregar o sistema, acelerando os possíveis indícios de envelhecimento de software), $2^{\circ}$ - Espera (para assegurar que o overhead realmente é oriundo do envelhecimento de software) e $3^{\circ}$ - Rejuvenescimento (para checar a efetividade do mecanismo de rejuvenescimento proposto). Um resumo dos resultados pode ser observados na Figura 1.

A primeira e segunda fase do experimento mostram que os efeitos percebidos são relacionados ao envelhecimento de software. A terceira fase mostra que a utilização de 
migração de máquinas virtuais é capaz de remover os efeitos acumulados pelo envelhecimento de software. Toda a descrição detalhada dos experimentos e resultados pode ser encontrada no Capítulo 3 da dissertação. Os resultados experimentais justificam a criação de modelos de disponibilidade para ambientes de nuvens que sofrem com o envelhecimento de software.

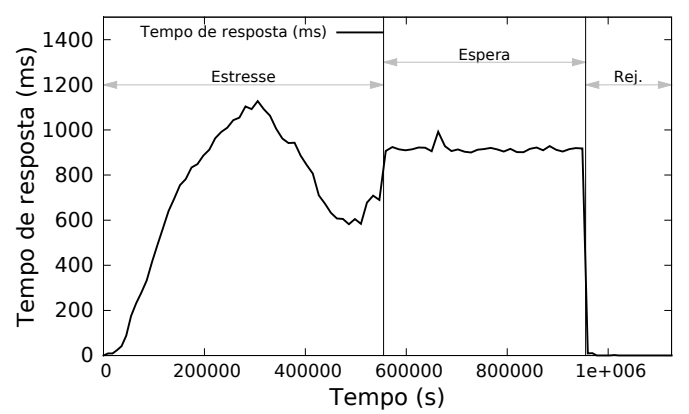

(a) Tempo de resposta (ms)

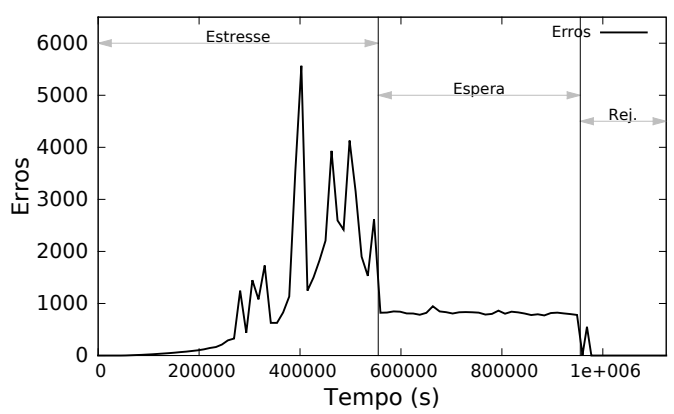

(b) Erros

Figura 1. Resumo dos resultados do experimento de envelhecimento de software

Após o término dos experimentos que detectaram o envelhecimento, é necessário dar início à construção dos modelos de disponibilidade para nuvens privadas. O primeiro conjunto de modelos proposto é baseado em RBDs e em CTMC [Maciel et al. 2012]. O intuito dos modelos iniciais é representar uma infraestrutura básica de computação em nuvem, composto por um FrontEnd, um nó e uma $V M$. Utiliza-se a abordagem hierárquica para a construção dos modelos, com o objetivo de reduzir a complexidade destes. Assim, blocos $R B D$ são empregados para representar o comportamento do FrontEnd e uma CTMC para representar o comportamento do nó que hospeda a $V M$. A partir de cada submodelo foram obtidas fórmulas fechadas para cálculo da disponibilidade. Ao fim, combinando as equações, obteve-se uma fórmula fechada para cálculo da disponibilidade do sistema por completo. Todo o procedimento pode ser encontrado na Seção 4.1 da dissertação.

Para aumentar a confiança nos resultados do modelo para infraestrutura básica de nuvem, decidiu-se validá-lo por intermédio de um processo baseado em injeção de falhas e reparos. Experimentos baseados nesse método foram realizados numa plataforma real. Foi necessário implementar um injetor de falhas e reparos para o ambiente, bem como utilizar ferramentas para monitorar o sistema. Os parâmetros utilizados nos scripts de teste foram obtidos de artigos já publicados [Melo et al. 2013]. Confrontando os resultados do monitoramento em ambiente real com os resultados obtidos dos modelos, é possível afirmar que o modelo construído realmente corresponde ao comportamento do sistema real. O procedimento é exibido na Seção 4.1 da dissertação.

Com o modelo da infraestrutura validado, a atividade seguinte é adaptá-lo a fim de que se passe a contemplar comportamentos de envelhecimento e rejuvenescimento de software. Assim, foi concebido outro modelo hierárquico capaz de representar tais comportamentos. Os modelos concebidos para os comportamentos de envelhecimento e rejuvenescimento foram baseados em $R B D$ e $S P N$ [Maciel et al. 2012]. O comportamento da migração da VM também é incluído no modelo, levando-se em consideração apenas o downtime causado por esta operação. Nesses modelos, a atividade de rejuvenescimento baseada em migrações obedece a um agendamento pré-definido. Existe um submodelo 
da rede que atua como um relógio, então quando o intervalo de tempo escolhido para o rejuvenescimento é alcançado, a migração está habilitada a ocorrer. Caso os outros prérequisitos da migração sejam satisfeitos, a migração da $V M$ ocorre, liberando assim o nó para o rejuvenescimento. O conjunto de modelos é apresentado na Seção 4.2 da dissertação. A partir da avaliação do modelos propostos é possível obter os agendamentos adequados para cada cenário proposto, e com isso maximizar a disponibilidade do sistema como um todo. A Figura 2 exibe o resultados de avaliação de um dos cenários estudados, o eixo Y corresponde à disponibilidade estacionária atingida e o eixo $\mathrm{X}$ corresponde ao tempo entre as migrações. Os resultados são comparados com cenários sem rejuvenescimento.

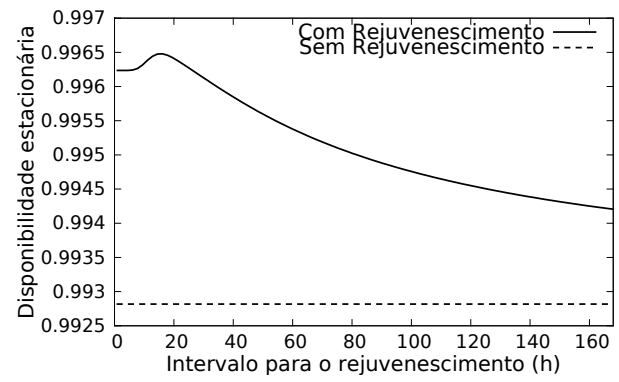

Figura 2. Resultados da avaliação dos modelos

A dissertação conta ainda com modelos de avaliação de disponibilidade com mecanismos de replicação baseada em cold-standby (Seção 5.3 da dissertação). E ainda é apresentada (na Seção 5.4 da dissertação) uma análise de sensibilidade no tempo gasto na migração em relação à disponibilidade do sistema.

\section{Conclusões}

A dissertação traz importantes avanços ao estado da arte em modelos de disponibilidade de ambientes de computação em nuvem com rejuvenescimento de software. O Capítulo 7 da dissertação possui as comparações com os trabalhos correlatos à dissertação. $\mathrm{O}$ trabalho apresentado na dissertação foi conduzido de modo a solidificar os resultados obtidos nos modelos finais. Os resultados constituem contribuições relevantes pois, trazem novas perspectivas para a área de estudo de modelos de disponibilidade em computação em nuvem, e ainda podem servir de insumo para gerentes de ambientes tomarem decisões administrativas nos ambientes de computação em nuvem. Além disso, podem-se destacar como contribuições dessa dissertação, que poderão ser amplamente reutilizados pela comunidade científica:

1. Conjunto de fórmulas fechadas para cálculo de disponibilidade de infraestruturas básicas de computação em nuvem;

2. Metodologia detalhada para validação de modelos de disponibilidade por intermédio de injeção de falhas;

3. Metodologia para condução de experimentos de envelhecimento de software em hypervisors e rejuvenescimento de software baseado em migração de máquinas virtuais;

4. Modelos de disponibilidade para nuvens privadas que consideram a presença de envelhecimento de software no hypervisor e rejuvenescimento de software baseado em migração de máquinas virtuais. 
A partir da pesquisa realizada três artigos foram publicados em anais de conferências.

1. Melo, M.; Maciel, P.; Araujo, J.; Matos, R.; Araujo, C., Availability study on cloud computing environments: Live migration as a rejuvenation mechanism, In Proceedings of the Dependable Systems and Networks (DSN), 2013 43rd Annual IEEE/IFIP International Conference on , vol., no., pp.1,6, 24-27 June 2013. DOI: 10.1109/DSN.2013.6575322; (Qualis CC: A1).

2. Melo, M.; Araujo, J.; Matos, R.; Araujo, C.; Maciel, P., Comparative Analysis of Migration-Based Rejuvenation Schedules on Cloud Availability. In: Proceedings of the 2013 IEEE International Conference on Systems, Man, and Cybernetics (IEEE SMC 2013). October 13-16, 2013 - Manchester, United Kingdom. DOI: 10.1109/SMC.2013.701; (Qualis CC: B2).

3. Torquato, M.; Araujo, J.; Maciel, P., Estudo Experimental de Envelhecimento de Software em Nuvens KVM/OpenNebula: Live Migration como Mecanismo de Suporte ao Rejuvenescimento de Software. In: XIII Workshop de Computação em Clouds e Aplicações (WCGA 2015), em conjunto com o $33^{\circ}$ Simpósio Brasileiro de Redes de Computadores e Sistemas Distribuídos (SBRC 2015), Disponível em http://sbrc2015.ufes.br/wpcontent/uploads/proceedingsWCGA20151.pdf. (Qualis CC: B4)

É importante salientar que existem alguns trabalhos para serem submetidos em veículos internacionais. As próximas submissões serão voltadas aos periódicos da área.

\section{References}

Araujo, J., Matos, R., Maciel, P., Matias, R., and Beicker, I. (2011). Experimental evaluation of software aging effects on the eucalyptus cloud computing infrastructure. In Proceedings of the Middleware 2011 Industry Track Workshop, page 4. ACM.

CISCO (2012). Cisco global cloud networking survey summary and analysis of results worldwide results. Technical report, CISCO.

Grottke, M., Matias, R., and Trivedi, K. S. (2008). The fundamentals of software aging. In Software Reliability Engineering Workshops, 2008. ISSRE Wksp 2008. IEEE International Conference on, pages 1-6. IEEE.

Huang, Y., Kintala, C., Kolettis, N., and Fulton, N. D. (1995). Software rejuvenation: Analysis, module and applications. In Fault-Tolerant Computing, 1995. FTCS-25. Digest of Papers., Twenty-Fifth International Symposium on, pages 381-390. IEEE.

Jain, R. (1991). The art of computer systems performance analysis - techniques for experimental design, measurement, simulation, and modeling. Wiley professional computing. Wiley.

Maciel, P. R. M., Trivedi, K. S., Jr., R. M., and Kim, D. S. (2012). Dependability Modeling, chapter 3. IGI Global.

Matias, R. et al. (2006). An experimental study on software aging and rejuvenation in web servers. In Computer Software and Applications Conference, 2006. COMPSAC'06. 30th Annual International, volume 1, pages 189-196. IEEE.

Melo, M., Maciel, P., Araujo, J., Matos, R., and Araujo, C. (2013). Availability study on cloud computing environments: Live migration as a rejuvenation mechanism. In Dependable Systems and Networks (DSN), 2013 43rd Annual IEEE/IFIP International Conference on, pages 1-6. 